\title{
sciendo
}

Current Issues in Pharmacy and Medical Sciences

Formerly ANNALES UNIVERSITATIS MARIAE CURIE-SKIODOWSKA, SECTIO DDD, PHARMACIA

journal homepage: http://www.curipms.umlub.pl/

\section{Peculiarities of the effects of a high-calorie diet on the structural components of the lymph nodes and under melatonin correction}

\author{
Tetiana Harapko ${ }^{1 \star}$ [D, Lesia MateshuK-VATSEBA ${ }^{2}[0$
}

${ }^{1}$ Department of Human Anatomy and Histology, Uzhhorod National University, Medical Faculty, Uzhhorod, Ukraine

${ }^{2}$ Department of Normal Anatomy, Lviv National Medical University named after Danylo Halytskyi, Lviv, Ukraine

\section{ARTICLE INFO \\ Received 17 January 2020 \\ Accepted 20 June 2020}

\section{Keywords:}

experiment,

lymph nodes,

sodium glutamate,

melatonine.

\begin{abstract}
Obesity is a chronic recurrent disease that is manifested by excess accumulation of adipose tissue and is a consequence of an imbalance in energy utilization and consumption in persons with or without hereditary predisposition. The purpose of the study is to study the morphometric and histological changes of the parenchyma of the lymph nodes of rats in experimental obesity and under the conditions of melatonin correction. The study was performed on 66 white rats of reproductive age. Microanatomy of the structural components of lymph nodes of white rats under physiological norms was examined in 10 intact animals. Experimental animals were divided into 4 groups. Eight weeks after the experimental animals were on a high calorie diet (HCD), there was a significant decrease in the relative area of the cortical substance in the parenchyma of the lymph nodes of white rats of males and females by $10.3 \%$ and $8.3 \%$, respectively, and an increase in the relative area of the medullary substance by $16.1 \%$ and $13.2 \%$, respectively, compared to an intact group of animals.

The relative area of the cortical substance in the parenchyma of the lymph nodes of white rats, which were for two weeks on HCD, then six weeks on HCD and melatonin, exceeds the parameters of the intact group of animals by only 3.2\% in males and 3.5\% in females. The relative area of the medullary substance is less than that of the intact group of animals by $5.0 \%$ in males and $5.5 \%$ in females. Under the conditions of melatonin correction it is found that the germinal centers of the secondary lymph nodes in the cortical substance are slightly increased. Trabecules extending from the capsule are clearly expressed and thickened, while arteries and arterioles show thickened walls and are full-blooded. Moreover, the veins are enlarged and full-blooded.
\end{abstract}

\section{INTRODUCTION}

One of the most pressing problems of modern society is being overweight or obese [1]. Obesity is a chronic recurrent disease that is manifested by excess accumulation of adipose tissue and is a consequence of the imbalance of energy utilization and consumption in persons with or without hereditary predisposition. Obesity is caused by following a highcalorie diet, and is often the outcome of adding sodium glutamate to food. Animal studies indicate that sodium glutamate can induce hypothalamic lesions and leptin resistance, possibly influencing energy balance, leading to overweight and obese $[2,3]$. It is a well-known fact that obesity causes

\footnotetext{
* Corresponding author

e-mail: garapkotv@gmail.com
}

a number of comorbidities and complications of the chronic course (hypertension, type 2 diabetes, atherosclerosis, some cancers, etc.) [4-6]. The cardiovascular system has been a major focus of research on obesity-induced pathology and recent studies have elucidated some of the mechanisms by which obesity promotes vascular injury. However, although the effects of obesity on the cardiovascular system have been intensely studied, there is comparably little known about how dietary changes or adipose accumulation impact the lymphatic system [7].

In general, the immune organs should also contribute to the onset of comorbidities caused by obesity, but very little is known what role the lymphatic system plays in the manifestation of obesity-related diseases [8-10]. Indeed, fat tissue 
has been found to produce a wide variety of "adipokines", involved in the regulation of numerous physiological functions, including the immune response [11].

We used melatonine to correct the changes caused by obesity. Melatonin is a molecule secreted by the pineal gland and is an important regulator of sleep and circadian rhythms [12-15]. It is also remarkably functionally diverse with actions as a free radical scavenger and antioxidant, circadian rhythm regulator, as well as an anti-inflammatory and immunoregulating molecule, and as an oncostatic agent [16]. Brown adipose tissue (BAT), for example, influences energy balance through nonshivering thermogenesis, and its metabolism daily and seasonal variations are regulated by melatonin through partially known mechanisms [17].

\section{AIM}

To study the morphometric and histological changes of the parenchyma of the lymph nodes of rats in experimental obesity and under the conditions of melatonin correction.

\section{MATERIALS AND METHODS}

We carried out the study on 66 white rats of reproductive age (2.5-6.5 months) weighing 120-280 g. Microanatomy of the lymph nodes structural components in white rats under conditions of physiological norm was studied on 10 intact animals (first group). Experimental animals were divided into 4 groups: the first group (10 animals), being fed a high-calorie diet (HCD) for eight weeks; the second group (10 animals), fed high-calorie diet for two weeks followed by two weeks of HCD and melatonin; the third group (10 animals), fed high-calorie diet for two weeks followed by four weeks of HCD and melatonin; the fourth group (10 animals), fed high-calorie diet for two weeks followed by six weeks of HCD and melatonin.

Each group included 5 male and 5 female rats. The applied high-calorie diet was achieved due to the fact that glutamate sodium was added into food in a dose of $0.07 \mathrm{~g} / \mathrm{kg}$ of rat body weight. The dose of melatonin was $10 \mathrm{mg} / \mathrm{kg}$ rat body weight, administered orally daily in the afternoon at the same time. Control was provided by 16 white rats fed a standard vivarium diet instead of a high-calorie diet.

All experimental animals were kept under the vivarium of the Danylo Halytsky Lviv National Medical University. The study was performed in accordance with the provisions of the European Convention for the protection of vertebrate animals used for experimental and other scientific purposes (Strasbourg, 1986), Council of Europe Directives 86/609/ EEC (1986), Law of Ukraine No. 3447-IV "On the Protection of Animals from Cruelty", the general ethical principles of experiments on animals adopted by the First National Congress of Ukraine on Bioethics (2001).

Images from the histological preparations of the mesenteric lymph nodes in the computer monitor were displayed from the MICROmed SEO SCAN microscope by means of the Vision CCD Camera. The studies were carried out within the established schedule of the trial in samples stained with hematoxylin, eosin and azane. Morphometric studies were performed on a personal computer by using VideoTest-5.0,
KAARA Image Base, Stepanizer and Microsoft Excel. Statistical processing of digital data was achieved using "Excel" and "STATISTICA" 6.0 software, using the parametric method.

\section{RESULTS}

In animals of the intact and control groups, according to our histological studies, the structure of the iliac and mesenteric lymph nodes was compliant with the species norm. Externally, the lymph nodes are surrounded by a connective-tissue capsule, from which numerous trabeculae lead inside the node's parenchyma. On the node's concave side, a hilum is located. Parenchyma consists of a cortical substance located on the node's periphery, and is closer to the gate of the medullary substance. Under the capsule there is a marginal sinus. In the cortical substance there are primary and secondary lymphoid follicles. The secondary ones contain germinal (clearing) centers surrounded by a marginal layer. The medullary substance contains lymph cords and medullary intermediate lymph sinuses (Fig. 1).

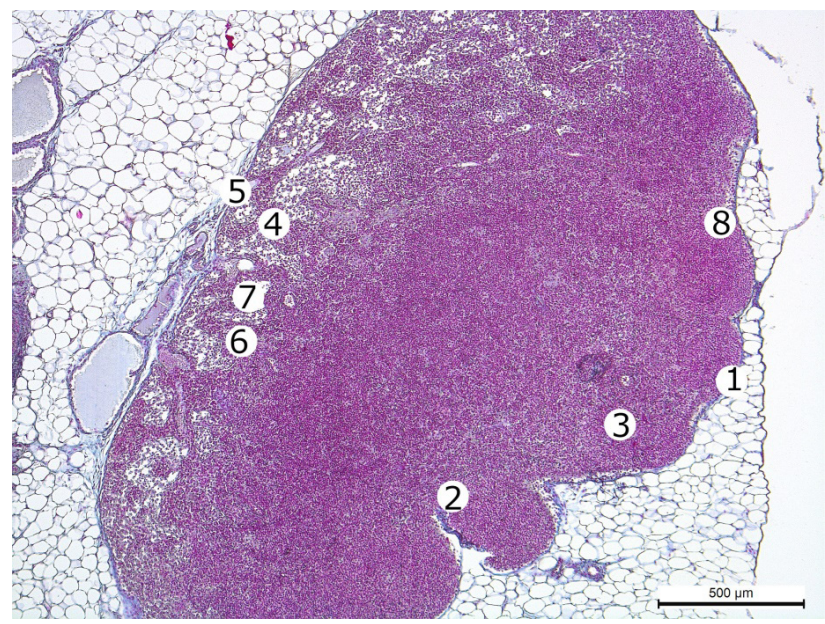

Azane stained. Magnif: obj. $\times 5$, ocul. $\times 10$.

Designation: 1 - capsule; 2 - trabecula; 3 - cortical substance

4 - medullary substance; 5 - the hilum of the node; 6 -lymph cord

7 - medullary lymph sinus 8 - marginal sinus

Figure 1. A mesenteric lymph node of an intact white rat male

Eight weeks after HCD, there is a significant decrease in the relative area of cortical substance in the parenchyma of lymph nodes of white rats of males and females to $54.8 \pm 1.6 \%$ and $56.12 \pm 1.65 \%$. These figures are $10.3 \%$ and $8.3 \%$ less than the parameters of the intact group of animals (see Tables 1,2). Accordingly, the relative area of the medullary substance increases to $45.2 \pm 0.87 \%$ in male rats, and to $43.88 \pm 0.78 \%$ in female rats. Such outcomes are $16.1 \%$ and $13.2 \%$ higher than the parameters of the intact group of animals (see Tables 1,2). Moreover, CMI was found to have decreased in both male and female rats by $22.9 \%$ and $19.0 \%$, respectively.

After 8 weeks of experiment, in both male and female rats, the medullary lymphatic sinuses expanded and deformed, the proportion of reticular connective tissue increased and the proportion of lymphocytes in their lumen decreased. B-lymphocytes, plasmocytes and macrophages were also found to be densely located in the medullary lymph cords. Often there were "empty" blood capillaries 
Table 1. Dynamics of changes in the relative areas of cortical substance, medullary substance and cortical-medullary index (CMI) of lymph nodes of white male rats of control and experimental groups $(\mathrm{M} \pm \mathrm{m})$

\begin{tabular}{|l|c|c|c|}
\hline \multicolumn{1}{|c|}{ Group name } & $\mathrm{S}_{\text {cortic.subst. } \%} \%$ & $\mathrm{~S}_{\text {medul.subst. } \%} \%$ & CMI \\
\hline Intact animals & $61.08 \pm 1.56$ & $38.92 \pm 0.78$ & $1.57 \pm 0.11$ \\
\hline I group - 8 weeks HCD & $54.8 \pm 1.6$ & $45.2 \pm 0.87$ & $1.21 \pm 0.2$ \\
\hline $\begin{array}{l}\text { II group - 2 weeks HCD, } \\
\text { 2 weeks HCD +melatonin }\end{array}$ & $65.93 \pm 1.56$ & $34.07 \pm 0.8$ & $1.94 \pm 0.16$ \\
\hline $\begin{array}{l}\text { III group - 2 weeks } \\
\text { HCD, 4 weeks } \\
\text { HCD+melatonin }\end{array}$ & $64.31 \pm 1.48$ & $35.69 \pm 0.67$ & $1.8 \pm 0.12$ \\
\hline $\begin{array}{l}\text { IV group - 2 weeks } \\
\text { HCD, 6 weeks } \\
\text { HCD+melatonin }\end{array}$ & $63.01 \pm 1.6$ & $36.99 \pm 0.76$ & $1.73 \pm 0.09$ \\
\hline
\end{tabular}

Table 2. Dynamics of changes in the relative areas of cortical substance, medullary substance and cortical-medullary index (CMI) of lymph nodes of white female rats of control and experimental groups $(\mathrm{M} \pm \mathrm{m})$

\begin{tabular}{|l|c|c|c|}
\hline \multicolumn{1}{|c|}{ Group name } & $\mathrm{S}_{\text {cortic.subst. }}(\%)$ & $\mathrm{S}_{\text {medul.subst. }}(\%)$ & CMI \\
\hline Intact animals & $61.23 \pm 1.7$ & $38.77 \pm 0.76$ & $1.58 \pm 0.11$ \\
\hline I group - 8 weeks HCD & $56.12 \pm 1.65$ & $43.88 \pm 0.78$ & $1.28 \pm 0.1$ \\
\hline $\begin{array}{l}\text { II group - 2 weeks HCD, } \\
\text { 2 weeks HCD+melatonin }\end{array}$ & $66.19 \pm 1.3$ & $33.81 \pm 0.9$ & $1.96 \pm 0.15$ \\
\hline $\begin{array}{l}\text { III group - 2 weeks } \\
\text { HCD, 4 weeks } \\
\text { HCD +melatonin }\end{array}$ & $65.83 \pm 1.23$ & $34.17 \pm 0.89$ & $1.93 \pm 0.2$ \\
\hline $\begin{array}{l}\text { IV group - 2 weeks } \\
\text { HCD, 6 weeks } \\
\text { HCD+melatonin }\end{array}$ & $63.35 \pm 1.4$ & $36.65 \pm 1.0$ & $1.73 \pm 0.09$ \\
\hline
\end{tabular}

with a thickened wall. In addition, in the paracortical area of the mesenteric lymph nodes, the number of postcapillary venules with high endothelium was found to have increased (Fig. 2).

The large number of lymphocytes in the wall and their lumen may indicate an increase in the processes of lymphocyte migration into the parenchyma of the lymph node from the blood. The latter confirms the common belief among morphologists that obesity is a chronic inflammatory process that leads to the continued activity of the immune protection units.

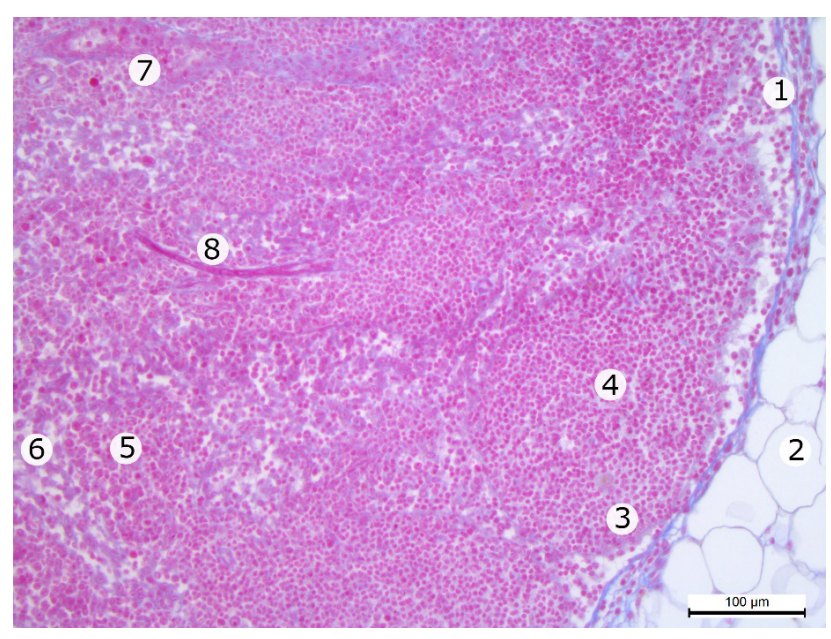

Stained with azan. Magnif: obj. $\times 10$, ocul. $\times 10$.

Designation: 1 - enlarged marginal sinus; 2 - adipose tissue in the thickness of the capsule and around the lymph node; 3 - secondary lymphoid follicles; 4 - germinal center of the lymphoid follicles; 5 - Billroth cords;

6 - medullary lymph sinus; 7 - postcapillary venule with high endothelium; 8 - blood capillary

Figure 2. A fragment of the lymph node of a white rat male after eight weeks of HCD
After 8 weeks of the experiment, we saw an increase in the number of secondary lymphoid follicles in the cortical substance of lymph nodes of white rats. In addition, germinative centers were expanded and enlightened, while a decrease in the relative area of the cortical area was seen. Moreover, arteries and veins were deformed and enlarged. Furthermore, vessels of the hemomicrocirculatory bed with damaged walls were often observed - this effect leads to hemorrhage in the parenchyma of the organ.

Morphometric indices in the second group of animals (two weeks of HCD, followed by two weeks of HCD + melatonin) indicate that the relative area of the cortical substance in the parenchyma of the lymph nodes of white rats of males and females increased by $7.9 \%$ and $8.1 \%$ compared to the intact group of animals, and is $65.93 \pm 1.56 \%$ and $66.19 \pm 1.3 \%$ (see Tables 1, 2). Accordingly, the relative area of the medullary substance decreases to $34.07 \pm 0.8 \%$ in male rats and $33.81 \pm 0.9 \%$ in female rats. These figures are $12.5 \%$ and $12.8 \%$ less than the parameters of the intact group of animals (see Tables 1,2). CMI in both male and female rats was $23.6 \%$ and $24.0 \%$, respectively - greater than the intact animal group parameter.

Histologically, in both male rats and female rats of the second experimentally group of animals, the overall structure of the lymph node corresponds to the intact group of animals. There is a slight enlargement of the marginal and cortical lymphatic sinuses (Fig. 3). Full-blooded veins and arteries also occurred. A somewhat thickened capsule was seen.

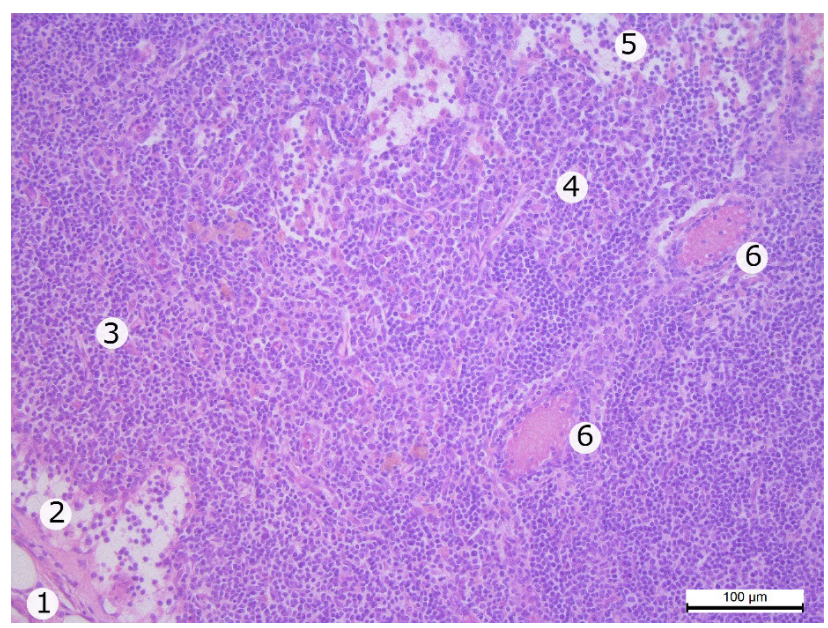

Stained with hematoxylin and eosin. Magnif: obj. $\times 20$, ocul. $\times 10$. Designation: 1 - capsule; 2 - marginal sinus; 3 - cortical substance; 4 - the paracortical area; 5 - medullary substance; 6 - enlarged and fullblooded artery

Figure 3. A fragment of the lymph node of the white rat male after two weeks HCD, followed by two weeks HCD + melatonin

Morphometric indices in the third group of animals (two weeks of HCD, followed by four weeks of HCD + melatonin) indicate that the relative area of the cortical substance in the parenchyma of lymph nodes of white rats of males and female rats decreased by $2.5 \%$ and $0.5 \%$ compared to the previous group of animals and were $64.31 \pm 1.48 \%$ and $65.83 \pm 1.23 \%$, respectively. These figures are $5.3 \%$ and $7.5 \%$, respectively, higher than those of intact animals (see Tables 1,2). Accordingly, the relative area of the medullary substance in the parenchyma of lymph nodes of white rats of males and females was found to have increased by $4.8 \%$ 
and $0.8 \%$ compared with the previous group of animals and is $35.69 \pm 0.67 \%$ and $34.17 \pm 0.89 \%$, which is $8.3 \%$ and $11.9 \%$ less than the intact animal parameter (see Tables 1, 2). CMI in both male and female rats was $14.6 \%$ and $22.2 \%$, respectively - greater than the intact animal group parameter.

On histological preparations of lymph nodes in both male rats and female rats of the third and fourth experimental groups of animals, the germinal centers of the secondary lymph nodes in the cortical substance were seen to have slightly increased. In addition, trabecules extending from the capsule were clearly expressed and thickened. What is more, arteries and arterioles had thickened walls and were full-blooded (Fig. 4). The veins were also enlarged and full-blooded. Moreover, the marginal sinus was unevenly expanded, the medullary lymphatic sinuses were enlarged and tortuous (Fig. 4), while B-lymphocytes, plasmocytes and macrophages were densely located in the medullary cords.

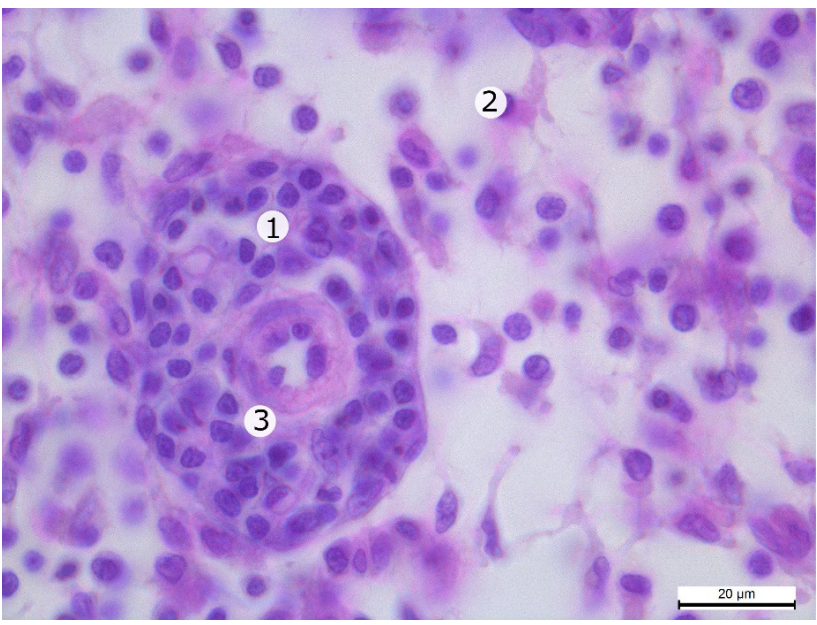

Stained with hematoxylin and eosin. Magnif: obj. $\times 100$, ocul. $\times 10$. Designation: 1 - medullary cords; 2 - medullary lymphatic sinus; 3 - arterioles with thickened wall

Figure 4. A fragment of the medullary substance of the lymph node of the white rat female after two weeks of HCD, followed by four weeks of $\mathrm{HCD}+$ melatonin

Morphometric indices in the fourth group of animals (two weeks of HCD, followed by six weeks of $\mathrm{HCD}+$ melatonin) indicate that the relative area of the cortical substance in the parenchyma of lymph nodes of white rats of males and females decreased by 2.0 and $3.8 \%$, respectively, to that of the previous group of animals and was $63.01 \pm 1.6 \%$ and $63.35 \pm 1.4 \%$. These indicators are $3.2 \%$ and $3.5 \%$ higher than the parameters of the intact group of animals (see Tables 1,2). Accordingly, the relative area of the medullary substance was found to have increased by $3.6 \%$ and $7.3 \%$ compared with the previous group of animals and was $36.99 \pm 0.76 \%$ in male rats and $36.65 \pm 1.0 \%$ in female rats. These figures are $5.0 \%$ and $5.5 \%$ less than the parameters of the intact group of animals (see Table 1). CMI in both male and female rats was $8.3 \%$ and $9.5 \%$, respectively, greater than the intact animal parameter.

\section{DISCUSSION}

Obesity has significant negative effects on lymphatic transport, dendritic cells (DC) migration, and lymph node architecture. Loss of $\mathrm{T}$ and $\mathrm{B}$ cell inflammatory reactions does not protect from impaired lymphatic fluid transport, but preserves DC migration capacity. Future studies are needed, however, to determine how the interplay between diet, obesity, and the lymphatic system modulate systemic complications of obesity. Still, we showed that diet induced obesity (DIO) in mice results in significant impairment of the lymphatic system as reflected by decreased lymphatic flow, changes in lymph node architecture, and impaired dendritic cell migration. Using a variety of techniques, we found that DIO results in impaired lymphatic transport in dermal lymphatics and drainage to the regional lymph nodes [7].

To examine the relation between increased adiposity and secondary lymph node morphology and immune cell populations, we utilized a Westernized diet to induce obesity in mice. Obesity caused reactive lymphoid hyperplasia, a change that was most prominent in the visceral lymph nodes. Increased visceral lymph node size was associated with increased viable cell number, which included increased numbers of pro- inflammatory antigen presenting cells, including but not limited to macrophages, dendritic and $\mathrm{T}$ helper cells. However, T regulatory cells were reduced in the visceral lymph nodes. Moreover, immune cell alterations in subcutaneous lymph nodes were limited solely to reductions in $\mathrm{T}$ regulatory cells. The visceral adipose depot also had greater reactivity towards HFD than subcutaneous. Principal HFD-induced changes in visceral adipose tissue occurred via shifts in relative immune cell populations leading to a greater percentage of macrophages, dendritic cells and CD8+ T cells. HFD has a greater influence on visceral cavity than the subcutaneous. In the visceral lymph node, but not subcutaneous, HFD- induced obesity decreased cell populations that suppressed immune function and increased those that regulate/activate immune response [8].

Our work indicates that obesity reduces the size of inguinal lymph nodes, impairs lymphatic fluid transport and migration of dendritic cells to peripheral lymph nodes, and reduces the number of $\mathrm{T}$ lymphocytes in lymph nodes. In general, obesity disrupts the integrity of the immune system and leads to changes in the development of leukocytes, their migration and diversity [3].

Decreasing the population of "naive" $\mathrm{T}$ cells (helper) leads to a deterioration of the immune system, which occurs with the onset of age. In animal model studies, obesity compromises the T-cell immune system due to increased adipogenesis in primary lymphoid organs and systemic inflammation. Due to the fact that obesity increases the risk of multiple age-related diseases, impaired immune competence is a possible mechanistic link between obesity and the development of diseases in the elderly [18-20].

Obesity-related insulin resistance is a chronic inflammatory condition that often gives rise to type 2 diabetes (T2D). Much evidence supports a role for pro-inflammatory $\mathrm{T}$ cells and macrophages in promoting local inflammation in tissues such as visceral adipose tissue (VAT) leading to insulin resistance. More recently, B cells have emerged as an additional critical player in orchestrating these processes. B cells infiltrate VAT and display functional and phenotypic changes in response to diet-induced obesity. B cells also contribute to insulin resistance by presenting antigens to $\mathrm{T}$ cells, secreting inflammatory cytokines, and producing pathogenic 
antibodies. B cell manipulation represents a novel approach to the treatment of obesity-related insulin resistance and potentially to the prevention of T2D [21].

The neuroimmunomodulatory effect of melatonin on the immune system is supported by the existence of specific melatonin receptors in immune organs, as well as immunocompetent cells; these melatonin receptors are located both in the plasma membrane and in the nucleus of the cells. The antioxidant properties of melatonin and its effects on neutrophil infiltration have been investigated in numerous experimental animal models. Peritoneal leukocytes were increased, for example, after an intraperitoneal melatonin injection in rats [22].

Melatonin regulates glucose metabolism by inducing nocturnal insulin resistance and diurnal insulin sensitivity, which is closely associated with nocturnal fasting and diurnal feeding. Melatonin regulates energy homeostasis, influencing feeding, and energy storage and expenditure. Melatonin tips the energy balance in the direction of reducing food intake and increasing brown adipose tissue energy expenditure, preventing excessive body weight gain [23].

\section{CONCLUSIONS}

As a result of the study performed on male and female rats, we found that:

1. After eight weeks of HCD, there was a significant decrease in the relative area of the cortical substance in the parenchyma of the lymph nodes of white rats of males and females by $10.3 \%$ and $8.3 \%$, respectively, and an increase in the relative area of the medullary substance by $16.1 \%$ and $13.2 \%$ respectively, compared to an intact group of animals.

2. After two weeks of HCD, followed by six weeks of $\mathrm{HCD}+$ melatonin, the relative area of cortical substance in the parenchyma of lymph nodes of white rats of males and females increased by $3.2 \%$ and $3.5 \%$ more than the parameters of intact group of animals. Accordingly, the relative area of the medullary substance is 5.0\% and 5.5\% less than the parameters of the intact group of animals.

3. Under the conditions of melatonin correction, we found that the germinal centers of the secondary lymph nodes in the cortical substance are slightly increased. Trabecules extending from the capsule are clearly expressed and thickened, while arteries and arterioles have thickened walls and are full-blooded. In addition, the veins are enlarged and full-blooded.

\section{AUTHORS' STATEMENT}

We do not declare any conflict of interests that could affect the objectivity and credibility of the work

\section{ORCID iDs}

Tetiana Harapko (1)https://orcid.org/0000-0003-0596-9622 Lesia Mateshuk-Vatseba (1)https://orcid.org/0000-0002-3466-5276

\section{REFERNCES}

1. Andersen CJ, Murphy KE, Fernandez ML. Impact of obesity and metabolic syndrome on immunity. Adv Nutr. 2016;7(1):66-75.
2. He K, Zhao L, Daviglus ML, Dyer AR, Horn LV, Garside D, et al. Association of monosodium glutamate intake with overweight in Chinese adults: the INTERMAP Study. Obesity. 2008;16(8):1875-80.

3. Beltiukova SV. Opredelenie glutamata natriia metodom tonkosloinoi khromatografii s liuminestsentnym detektirovaniem. Visnyk ONU. Khimiia. 2016;1(57):50-8.

4. Kothari V, Luo Y, Tornabene T, O’Neill AM, Greene MW, Geetha T, et al. High fat diet induces brain insulin resistance and cognitive impairment in mice. Biochim Biophys Acta. 2017;1863:499-508.

5. Wan H, Wu S, Wang J, Yang Y, Zhu J, Shao X, et al. Body mass index and the risk of all-cause mortality among patients with nonvalvular atrial fibrillation: a multicenter prospective observational study in China. Eur J Clin Nutr. 2017;71(4):494-9.

6. Guarner V, Rubio-Ruiz ME. Low-grade systemic inflammation connects aging, metabolic syndrome and cardiovascular disease. Interdiscip Top Gerontol. 2015;40:99-106.

7. Weitman ES, Aschen SZ, Farias-Eisner G, Albano N, Cuzzone DA, Ghanta S, et al. Obesity impairs lymphatic fluid transport and dendritic cell migration to lymph nodes. PLoS One. 2013;8(8):e70703.

8. Magnuson AM, Regan DP, Fouts JK, Booth AD, Dow SW, Foster MT. Diet-Induced obesity causes visceral, but not subcutaneous, lymph node hyperplasia via increases in specific immune cell populations. Cell Prolif. 2017;50(5):e12365.

9. Sato MN, Suzui M, Yoshino H, Kaburagi T, Sato K. Long term effects of high fat and sucrose diets on obesity and lymphocyte proliferation in mice. J Nutr Health Aging. 2009;13:602-6.

10. Suami H, Scaglioni MF. Lymphatic territories (lymphosomes) in the rat: an anatomical study for future lymphatic research. Plast Reconstr Surg. 2017;140(5):945-51.

11. Versini M, Jeandel PY, Rosenthal E, Shoenfeld Y. Obesity in autoimmune diseases: not a passive bystander. Autoimmun Rev. 2014;13:981-1000.

12. Yeh C-M, Su S-C, Lin C-W, Yang W-E, Chien M-H, Reiter RJ, et al. Melatonin as a potential inhibitory agent in head and neck cancer. Oncotarget. 2017;8(52):90545-56.

13. Buonfiglio D, Parthimos R, Dantas R, Silva RC, Gomes G, AndradeSilva J, et al. Melatonin absence leads to long-term leptin resistance and overweight in rats. Front Endocrinol (Lausanne). 2018;27(9):122.

14. Tan DX, Hardeland R, Back K, Manchester LC, Alatorre-Jimenez MA, Reiter RJ. On the significance of an alternate pathway of melatonin synthesis via 5-methoxytryptamine: comparisons across species. J Pineal Res. 2016;61:27-40.

15. Kalmykova O, Dzerzhynsky M. The effects of melatonin administration in different times of day on the brown adipose tissue in rats with high-calorie diet-induced obesity. Bull T Shevchenko Nat Univ Kyiv Ser Biol. 2019;77:55-61.

16. Manchester LC, Coto-Montes A, Boga JA, Andersen LP, Zhou Z, Galano A, et al. Melatonin: an ancient molecule that makes oxygen metabolically tolerable. J Pineal Res. 2015;59:403-19.

17. Souza CAP, Gallo CC, Camargo LS, Carvalho PVV, Olescuck IF, Macedo F, et al. Melatonin multiple effects on brown adipose tissue molecular machinery. J Pineal Res. 2019;66(2):e12549.

18. Yoshida K, Nakashima E, Kubo Y, Yamaoka M, Kajimura J, Kyoizumi S, et al. Inverse associations between obesity indicators and thymic T-cell production levels in aging atomic-bomb survivors. PLOS ONE. 2014;9(6):98-101.

19. Lobov GI, Pan'kova MN, Abdreshov SN. Transport function of the lymph nodes in young and old animals. Uspekhi Gerantol. 2015;28(4):681-6.

20. Demchenko GA, Abdreshov SN, Nurmakhanova BA. Contractile activity of lymph nodes in young, middle-aged, and old rats. Bull Exp Biol Med. 2019;67:194-7.

21. Winer DA, Winer S, Chng MH, Shen L, Engleman EG. B lymphocytes in obesity- related adipose tissue inflammation and insulin resistance. Cell Mol Life Sci. 2014;71:1033-43.

22. Calvo JR, Gonzalez-Yanes C, Maldonado MD. The role of melatonin in the cells of the innate immunity: a review. J Pineal Res. 2013; 55:103-20.

23. Do Amaral FG, Andrade-Silva J, Kuwabara W, Cipolla-Neto J. New insights into the function of melatonin and its role in metabolic disturbances. Expert Rev Endocrinol Metab. 2019;14(4):299-303. 